\title{
Pregnancy Outcome of a Patient Following Myomectomy Performed During First Trimester for Red Degeneration of Fibroid : A Case Report
}

\author{
Asma Habib ${ }^{1}$, Shahla Khatun ${ }^{2}$, Nafisa Khanum ${ }^{3}$ \\ ${ }^{1}$ Assistant Professor, ${ }^{2}$ Professor and Chair-Person, Department Of Obstetrics \& Gynaecology, ${ }^{3}$ Senior Consultant, Department of Anaesthesia, Bangla- \\ desh Medical College and Hospital
}

\begin{abstract}
:
Pain due to red degeneration of fibroid during pregnancy is usually associated with large myomas. Necrobiosis / red degeneration typically manifests itself about midpregnancy when the leiomyoma suddenly becomes acutely painful, enlarged and tender. The common differential diagnosis of this condition are torsion of the pedicle of an ovarian cyst/a leiomyoma, abruption placentae, acute pyelitis or any abdominal catastrophe. Ultrasound can easily delineate the presence of myomas of mixed echogenicity along with pregnancy and clinical findings usually suggest the diagnosis of pregnancy complicated by red degeneration of fibroid. The acute pain usually subsides within 3-10 days of conservative treatment. Only refractory cases ( $2 \%$ of patients) may demand surgical intervention in early gestation with the known risk of miscarriage. Here we report a pregnancy managed at 13 weeks by myomectomy for red degeneration. The patient ultimately delivered a healthy female child at 38 weeks by lower segment caesarean section.
\end{abstract}

Key words: early pregnancy, myomectomy, pregnancy outcome

[BSMMUJ $2013 ; 6(1): 69-74]$

\section{Introduction:}

Uterine myoma is the most common solid pelvic tumour occurring in $20-40 \%$ of women during their reproductive years. The reported prevalence of myomas in pregnancy is $2 \%$.Usually, a myoma during pregnancy remains asymptomatic but it can also cause spontaneous miscarriage, pain due to "red degeneration", preterm delivery, premature rupture of membranes, malpresentation, fetal compression syndrome, antepartum and postpartum bleeding, obstructed labour, retained placenta and uterine torsion. Sepsis can also be correlated with infected myomas. ${ }^{1,2}$ Malignant alteration of myomas is extremely rare, ranging between $0.13-0.29 \% .{ }^{3}$ One of the most common complications of myoma in pregnancy is the syndrome of "painful myoma". It is a direct result of degeneration occurring in 5-8\% of myomas in pregnancy as in our case at first trimester. Red degeneration of myoma usually responds to conservative treatment: opioid analgesics, tocolytic and empirical progesterone

Address for Correspondence: Dr. Asma Habib

House: 74 (502), Road no: 8/A, Dhanmondi Residential Area, Dhaka-1209

E-mail: asma.habib54@yahoo.com Mobile: 01712878035 therapy. 'According to Lolis et al indications for myomectomy during pregnancy include severe abdominal pain due to torsion of subserous pedunculated myomas or red degeneration not responding to medical treatment, and an increase in myoma size causing abdominal discomfort Myomectomy may be indicated when the pelvis is totally or partially blocked by a fibroid growing from the cervix or lower uterine segment. ${ }^{4}$ Here we report the outcome of a pregnancy complicated by red degeneration of fibroid at 13 weeks managed by myomectomy. She delivered a healthy female child weighing 3.00 kilograms at 37 completed weeks by caesarean section due to foetal distress.

\section{Case report:}

A 30 year old nulliparous woman, working as a care provider in a primary school presented with lower abdominal pain and extreme anxiety along with an urgent desire for removal of the abdominal mass as ultrasonogram revealed multiple fibroid uterus along with single intrauterine pregnancy corresponding to 8 weeks of gestation. Sonography reported gravid uterus with multiple hypoechoiec lesions of variable sizes, largest two of them 
The pelvicalyceal system of the left kidney also reverted to normal dimensions on subsequent sonographic followups. There were two newly forming fibroids, one in the fundus measuring $3 \mathrm{~cm} \times 3.5 \mathrm{~cm}$ and one near the lower segment on the anterior wall at 30 weeks sonography with an estimated foetal weight of 1.5 kilograms. The patient had good weight gain and could carry out her activities without complaints. At 34 weeks of pregnancy, a subserous fibroid could be felt on the fundus along with a corresponding symphysio-fundal height. The patient was counseled for an elective caesarean section after the completion of 37 weeks considering the risk of uterine dehiscence during labour, because during removal of the posterior intramural myoma there was gross thinning of the posterior wall. She had a cesarean section at 38 weeks as she experienced significant discomfort on carrying out daily activities along with less foetal movement. She delivered a female child of 3.00 kilograms. There was marked adhesion in the fundus with a $3 \mathrm{~cm}$ sub-serous fibroid along with a $3 \mathrm{~cm}$ intramural fibroid occupying the lower part of the body of the anterior uterine wall, which were kept untouched during c-section. The patient had a normal post-operative recovery and experienced a normal puerperium.

\section{Discussion:}

A large number of fibroids complicating pregnancy require no treatment. Pregnancy and labour progress undisturbed in a large number of cases. Where a fibroid during pregnancy is not causing symptoms, but occupies such a position that it is certain to obstruct labour, the pregnancy should be allowed to proceed and Caesarean section, followed by myomectomy or hysterectomy, may be done. ${ }^{2,3}$ The size of individual tumour, number of tumours and their anatomical position to the developing foetus affect the success of myomectomy and may determine the course of pregnancy. A single tumour not involving the lower uterine segment or cervix, and bulging on the peritoneal surface, can be easily and safely enucleated without disturbing the pregnancy; but usually operation is not indicated; as the pregnancy progresses and the tumour does not cause symptoms.

Myomectomy during pregnancy is a treatment option for rapidly growing myomas which provoke resistance to conservative therapy and produce significant distortion of endometrial cavity leading to abortion or fetal anomalies despite the known risk of abortion and hemorrhage due to increased vascularity of gravid uterus. ${ }^{4}$ However, hypercoagulability in pregnancy may contribute in achieving hemostasis.The risk of abortion following myomectomy during pregnancy is reported to be $18 \%$ to $35 \%$ cases. However Lolis et al reported that only one patient among 13 patients requiring surgical intervention due to symptomatic myomas aborted postoperatively. ${ }^{4,5}$

Between the 15th and 56th days of gestation, the human embryo is said to be most vulnerable to the teratogenic effects of a drug. ${ }^{7}$ Since 1978 , most drugs used in medicine and anaesthesia have been assigned codes in the Swedish Catalogue of Registered Pharmaceutical Specialities (FASS). These codes are a guide to the appropriate choice of agents with respect to effects on the fetus, placenta and utero-placental blood flow, and the possibility of induced abortion. Studies of outcomes in large numbers of women who underwent surgery during pregnancy suggest no increase in congenital abnormalities but a greater risk of abortion, growth restriction and low birth weight with a conclusion that these problems resulted from primary disease or the surgical procedure itself rather than exposure to anaesthesia. Although the data available are incomplete, studies suggest that the administration of a hypnotic, opioid analgesia or sedative drug will not have deleterious effects on embryonic or fetal development. Nitrous oxide was avoided as animal studies provided evidence that it is a potent inhibitor of methionine synthase. However, currently, there is no evidence in human beings to suggest that this is clinically significant and therefore can be used in general aneasthesia. $^{?}$

After 6-8 weeks gestation, the maternal cardiac, haemodynamic, respiratory, metabolic and pharmacological parameters are considerably altered. With the increase in minute ventilation and oxygen consumption and a decrease in oxygen reserve (decreased functional residual capacity and residual volume), pregnant women become hypoxaemic more rapidly. Therefore supplementary oxygen must always be given during anaesthasia to maintain oxygenation. Normal hyperventilation in pregnancy 
measuring about $10.2 \times 9.1 \mathrm{~cm}$ in the fundal region and $7.4 \times 6.6 \mathrm{~cm}$ seen in the anterior and left lateral aspect of the myometrium with moderate left sided hydronephrosis. Examination revealed uterine height corresponding to 20 weeks of gestation with nodularity felt over the fundus of the uterus .She was counseled for continuation of pregnancy upon empirical hormone supports with Inj. Hydroxy progesterone caproate twice weekly along with oral allylestrenol and bed rest. At 13 weeks of gestation she was admitted with severe pain and intolerable discomfort in the whole abdomen rendering her unable to walk properly or to get up from lying position. She had also developed difficulty in voiding with narrow stream and scanty micturation. Examination revealed uterine height corresponding to 28 weeks size with a mass of soft to firm consistency about $12 \mathrm{~cm}$ apparently springing from the fundus of the uterus with tenderness elicited on palpation and restricted mobility. The clinical diagnosis of red degeneration of fibroid in pregnancy with obstructive uropathy was entertained. The patient was determined to remove the uterus bearing the fetus if necessary to ensure removal of all tumours producing this intense pain and discomfort refractory to the empirical/conservative treatment. She had the fear of recurrence of the tumours if the uterus was preserved. We consulted with urologists to perform left sided uteretic stenting to relieve the compression on the left ureter by the myoma. She remained indifferent and did not give consent to the procedure. Her pain aggravated much despite intravenous opioid therapy. Only after counseling with assurance to remove as many tumours as feasible, she gave consent for laparotomy. The risk of spontaneous abortion after the procedure was explained to her, but she remained complacent as the decision of surgical removal of the tumours had been taken, as this would marked alleviate her pain and discomfort. Laparotomy was performed on 12/9/2011 by right paramedian incision extending $6 \mathrm{~m}$ beyond the umbilicus under general anaesthesia. Upon opening the peritoneal cavity, a grossly distorted and enlarged uterus was found. A huge subserous fibroid about $12 \mathrm{~cm}$ in length protruding from the right corneal region was found with a broad pedicle $(8 \mathrm{~cm})$. The free tip of that subserous fibroid was found necrosed with adhesion to the right hepatic flexure of the colon and surrounding small bowel.

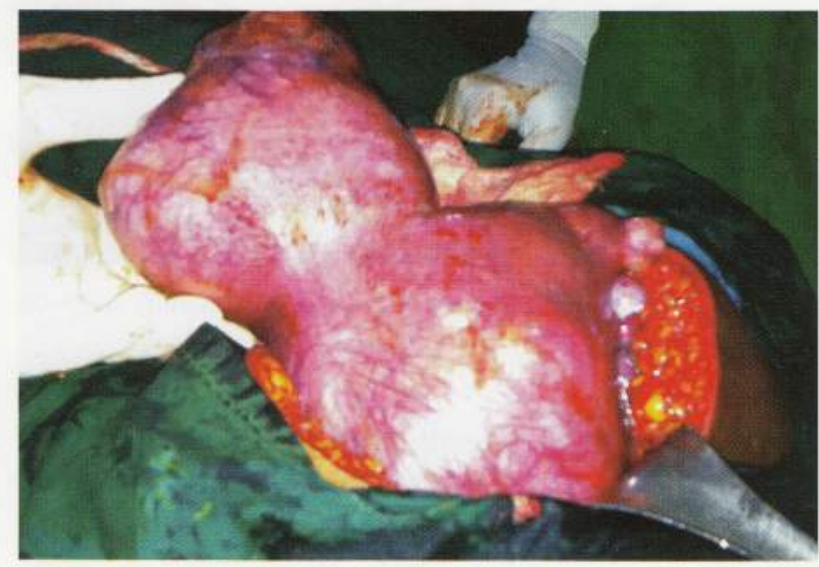

Fig-1.a: Laparotomy showing large sub-serous fibroid projecting from the fundus of the gravid uterus at 13 weeks.

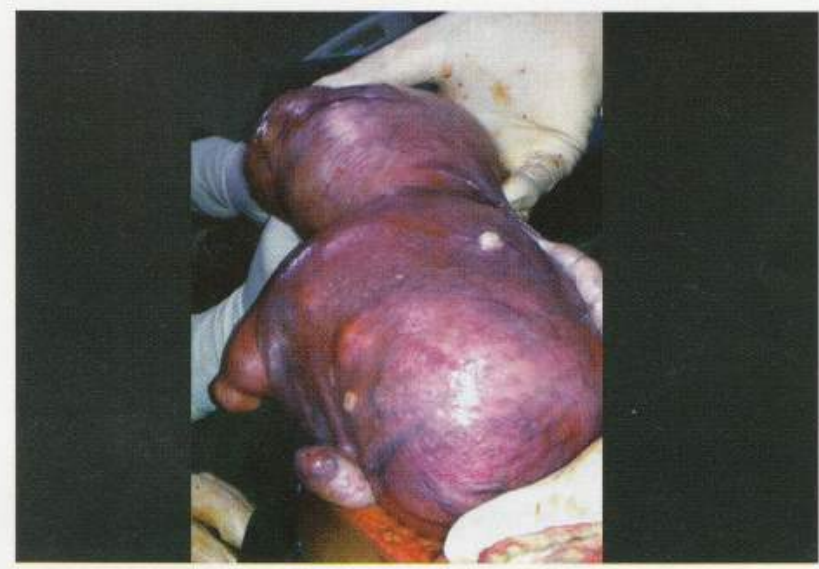

Fig-1.b: Laparotomy showing the posterior aspect of the uterus with a large intramural myoma along with the fundal fibroid.

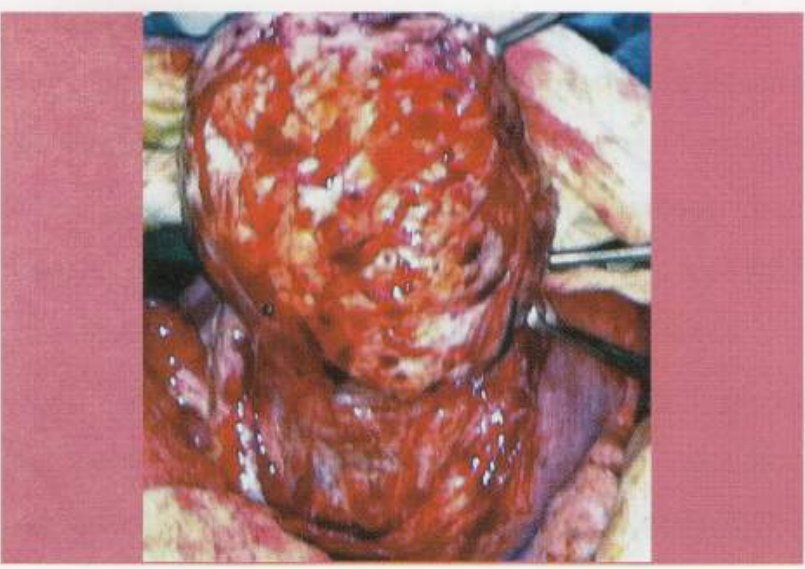

Fig-1.c: Extensive myometrial dissection during removal of the posterior myoma. 


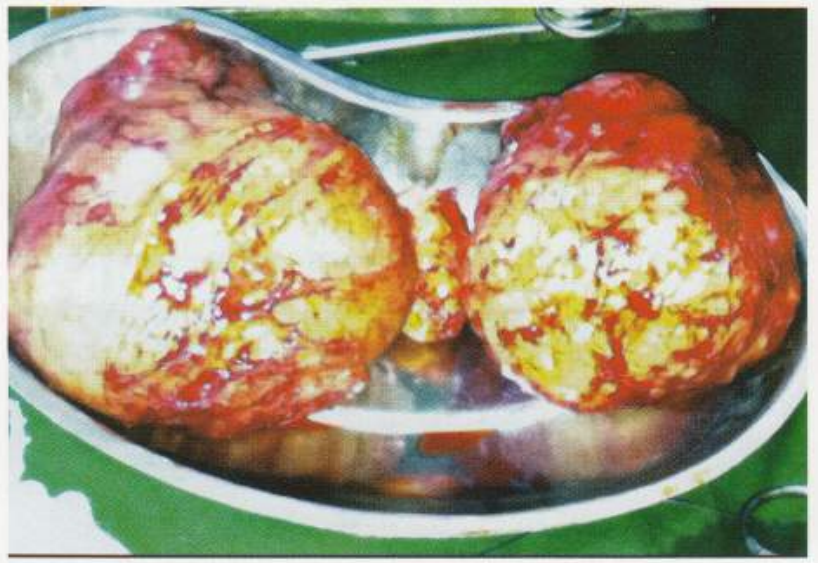

Fig-1.d: A kidney dish displaying the three successfully removed myomas from the gravid uterus.

(Fig-: 1. $a, b, c, d$ ) With gentle digital separation, the tip of the degenerated myoma was disengaged. The myoma was removed by incising the pedicle by electrocauterization followed by haemostatic ligature of the base with unremarkable bleeding. Upon further exploration, a large intramural round $(10 \times 10 \mathrm{~cm})$ myoma was found in the posterior wall of the body of the uterus almost impacted in the Pouch of Doulgas. A transverse posterior incision on the body of the uterus was made followed by gentle enucleation of the myoma along the cleavage plane. During separation the endometrial cavity was not opened. A thin but intact layer of deciduas was found to separate the anterior aspect of this posterior myoma from the gestational sac bearing the foetus. Myoma bed was closed by interrupted haemostatic sutures. A small isthmic anterior fibroid measuring $2.5 \mathrm{~cm}$ was found and attempt to enucleate it produced much bleeding which was ultimately controlled. As all incisions were haemostatically secured, no intraperitoneal drain was kept. The removed myomas measured $1600 \mathrm{gm}$ largest one, $900 \mathrm{gm}$ the second largest one and $200 \mathrm{gm}$ the smallest one, giving a total of 2700 gm of myoma mass removed. Histopathology of the myoma revealed red degeneration of leiomyomas as evidenced by ghosts of muscle cells and their nuclei and delicate off-pink staining of whorled interlacing myometrial fibers by fresh blood pigments.

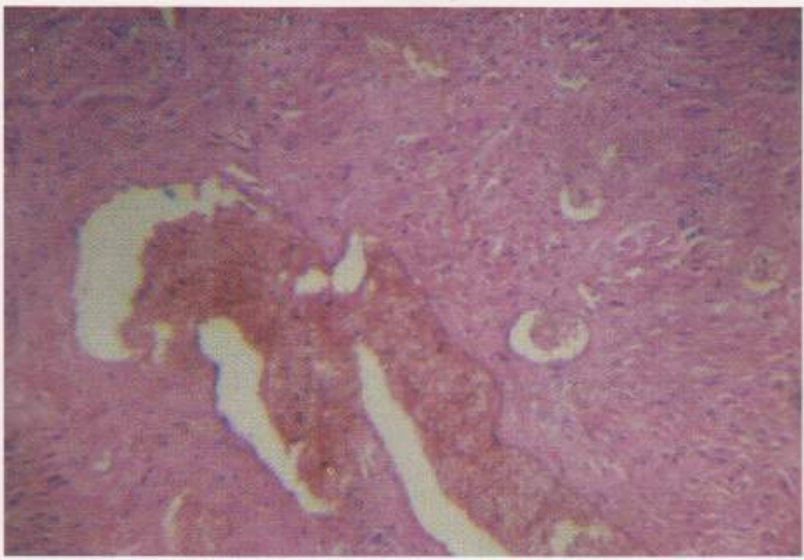

Fig-2: Histopathologic photography demonstrating red degeneration / necrobiosis of leiomyoma.

(Fig-: 2). The patient had relatively good post-operative convalescence as she experienced significantly less pain than before. She was put on progestogen hormone support along with analgesics. Foetal viability was reconfirmed on the following day of myomectomy and found to be satisfactory with good cardiac activity. The patient was advised to continue the Inj. Hydroxy progesterone caproate at weekly intervals upto 22 weeks followed by injections at 2 week interval upto 26 weeks onwards. Ultrasonography was advised to be repeated at 2 weeks interval to see foetal condition along with uterine growth/ any newly forming myomas. The patient was put on low dose antiplatelet therapy with aspirin $75 \mathrm{mg}$ up to 32 weeks. During subsequent antenatal checkup, she did not experience any significant recrudescence of pain except for one episode of dull ache at $20^{\text {th }}$ week of gestation which was managed conservatively. Serial ultrasosnography performed at 2 to 3 weeks interval from 14 weeks revealed satisfactory foetal growth corresponding to average percentile and no gross anomaly was detected. results in lowered expired $\mathrm{CO}_{2}(32-34 \mathrm{~mm} \mathrm{Hg})$ which should be maintained during anaesthesia. Pregnancy is associated with lower anaesthetic requirements, although the mechanism for this is unknown. ${ }^{7}$ The minimum alveolar concentration (MAC) for inhalation anaesthetics is reduced by $30 \%$ as early as $8-12$ weeks gestation. Intravenous (I.V) drugs that induce general anaesthesia should also be given in lower doses. Fetal well-being should be assessed by ultrasound or Doppler before and after anaesthesia and surgery. Because of the increased risk of hypoxaemia, difficulties with intubation, acid aspiration and risks to the fetus, regional anaesthesia should be 
selected over general anaesthesia whenever feasible. ${ }^{7.8}$ However this patient tolerated general anaesthesia without difficulty with a swift reversal. Serial Ultrasonography during pregnancy may be useful in monitoring fetoplacental growth and evaluating any residual myoma size following myomectomy during pregnancy, which was carried out during this particular case. It helped to evaluate growth of the foetus, placental localization, any newly developing myomas.

Proluton Depot (hydroxyprogesterone caproate) is indicated for prophylaxis of abortion in high risk pregnancies because it compensates for the hormone deficit, induces quiescence of the uterus and stimulates growth of an underdeveloped uterus. ${ }^{9}$ In this case, it was administered three times weekly $150 \mathrm{mg}$ from 11 weeks up to 27 weeks of gestation empirically.

Myometrial recontruction technique following myomectomy has been seen to affect the integrity of the uterus in subsequent pregnancy. After extensive dissection of myometrium during myomectomy with deep penetration of the uterine wall, caesarean section is the recommended method of delivery. ${ }^{10,11}$ Pregnancy following any surgical procedure involving the uterus has an increased risk of rupture or dehiscence during pregnancy and labor. The risk of uterine dehiscence in relation to cesarean sections has been well quantified, but the same cannot be applied in cases of women with a previous history of myomectomy, whether open or laparoscopic, because of the absence of good quality studies. One possible cause of uterine rupture after laparoscopic myomectomy is the wide use of electrosurgery that may result in poor vascularisation and tissue necrosis with an adverse effect on scar strength. ${ }^{11,12}$ Excess thermal damage should be avoided and adequate uterine repair must be assured using multiple layer suturing techniques. Both thermal damage and hematoma formation have been blamed as causes for suboptimal healing and rupture during a future pregnancy (Dubuisson et al, 2000; Landi et al, 2003). ${ }^{12,13}$ The unidirectional barbed wire suture with intracorporeal knots is the latest advancement of laparoscopic myomectomy technique. ${ }^{14}$ In a series of 13 patients who underwent myomectomy during pregnancy, the average gestational age was found to be 16 weeks and the weight of the largest myoma removed ranged from 105 to 2274 gram. Except for 2 cases (one ending in spontaneous abortion at 15 weeks and another having preterm labour at 29 weeks ) 11 cases delivered at 37 weeks or beyond with an average birth weight of $3000 \mathrm{gm}$. In this case, myomectomy was performed at 13 weeks and the largest removed myoma weighed about 1600 grams. $^{15,16}$ Histopathological findings of reported cases revealed various forms of degeneration (hyaline, red, cystic degeneration with foci of haemorrhagic infarction) along with torsion of the leiomyoma ${ }^{17}$ This particular case demonstrated typical diffuse salmon pick staining of the tumour with thrombosis in the vessels.

\section{Conclusion:}

Myomas are often encountered during pregnancy but the role of myomectomy during pregnancy is still debatable. Pain producing myomas usually respond to conservative treatment. Atypically large myomas refractory to non-surgical maneuvers may require surgical removal to relieve pressure symptoms. Review of carefully selected cases of myomectomy in early pregnancy has revealed both good maternal and foetal outcome. The anaesthetic risks have to be minimized as much as feasible. Optimum analgesia and empirical hormone therapy should be ensured in the immediate post-operative period. The antenatal period subsequent to myomectomy requires utmost vigilance and surveillance for the resurgence of pain in any remaining or newly forming myomas.These measures may ultimately lead to a successful reproductive outcome.

\section{References:}

1. Wallach EE, Vlahos NF. Uterine myomas: an overview of development, clinical features, and management. Am Coll Obstet Gynecol 2004; 104:393-406.

2. Phelan JP. Myomas and pregnancy. Obstet Gynaecol Clin North Am $1995 ; 22: 801-5$.

3. Neerja Bhatla, Biswa Bhushan Dash, Alka Kriplani and Nutan Agarwal. Myomectomy during pregnancy: A feasible option. The journal of obstetrics and gynaecology research (2009); 35( 1): 173-175

4. Krepelka P, Herman H. 2200 g-weight-fibroid: abdominal myomectomy in the twentieth week of pregnancy. Ceska Gynekol.2008 Oct; $73(5): 289-93$. 
5. Lolis DE, Kalantaridou SN, Makrydimas G, Sotiriadis A, Navrozoglou I, Zikopulos K, Paraskevaidis EA. Succesful myomectomy during pregnancy. Hum Reprod 2003; 18:1699-1702.

6. Andrijana Müller Vranješ, Siniša Šijanović, Domagoj Vidosavljevié et al. Surgical treatment of large smooth muscle tumor of uncertain malignant potential during pregnancy. Medicinski Glasnik, , 2011; $8(2): 290-292$

7. Nina Kylie Dorothy Walton. Anaesthesia for non-obstetric surgery during pregnancy. Contin Educ Anaesth Crit Care Pain (2006) 6 (2): 83-85.

8. Umezurike C, Feyi-Waboso P. Succesful myomectomy during pregnancy: case report. Reprod Health 2005; $2: 6$.

9. Frederick J. Mccann. Pregnancy complicated by a Fibroid Tumour showing "Red Degeneration." Proc R Soc Med. 1908 ;1 ( Obstet Gynaecol Sect): $315-320$

10. Myomectomy and its impact on fertility and reproductive outcome. JPOG MAY/JUN 2008・116

11. Hanom Husni Syam. Pregnancy Outcomes Following Laparoscopic Myomectomy. World Journal of Laparoscopic Surgery, JanuaryApril 2008;1(1):35-40
12. Dubuisson J, Fauconnier A, Deffarges J, Norgaard C, Kreiker G and Chapron C. Pregnancy outcomè and deliveries following laparoscopic myomectomy. Hum Reprod 2000;15,869-73.

13. Khalil E Rajab, Eftikhar Al- Ojaimi,Sundari M. Spontaneous Uterine Rupture in the Second Trimester of Pregnancy Associated with Red Degeneration of Fibroid. Bahrain Medical Bulletin, Vol. 28, No. 1, March 2006

14. Dubuisson JB, Chavet X, Chapron C, et al. Uterine rupture during pregnancy after laparoscopic myomectomy. Hum Reprod $1995 ; 10,1475-7$.

15. Nehzat F, Seidman DS, Nehzat C, et al. Laparoscopic myomectomy today: why, when and for whom? Hum. Reprod 1996;11,933-4.

16. Paul PG, Koshy AK, Thomas T. Pregnancy outcomes following laparoscopic myomectomy and single-layer myometrial closure. Hum Reprod 2006;21(12),3278-81.

17. Seracchioli R, Manuzzi L, Vianello F, et al. Obstetric and delivery outcome of pregnancies achieved after laparoscopic myomectomy. Fertil Steril 2006;86(1),159-65. 